\title{
Erratum: Landau-Zener extension of the Tavis-Cummings model: Structure of the solution [Phys. Rev. A 94, 033808 (2016)]
}

Chen Sun and Nikolai A. Sinitsyn

(Received 10 June 2017; published 26 June 2017)

DOI: 10.1103/PhysRevA.95.069907

In our original paper we made a typographical error in Eq. (50). The $\log x$ in that equation should be replaced by $-\frac{1}{\log x}$. So Eq. (50) should read as follows:

$$
n_{b}=2 S-\frac{1}{\log x}[\log (-\log x)-\gamma] \text { for } g>\frac{1}{2 S} .
$$

No other results are influenced by this typographical error, including Fig. 3(a) with a plot for this formula. 